\title{
Behaviour of impact oscillator with soft and preloaded stop
}

\section{František Peterka}

\author{
Institute of Thermomechanics, Academy of Sciences of the Czech Republic Dolejss`kova 5, 18200 Prague 8, Czech Republic
}

New phenomena of the dynamics of oscillators with impacts, when the stiffness of the stop changes from zero to infinity, are described. Dynamics of one example of the system with soft impacts, as the model of the piercing machine, is explained in more detail by bifurcation diagrams, time series, phase trajectories and Poincarè maps of periodic and chaotic impact motions. Optimal combinations of system parameters are found for the obtaining of maximum beforeimpact velocities.

\section{Introduction}

This work considers the dynamics of oscillators with soft impacts, see Fig. 1(b). Soft in this context is used for impacts where the duration of impact is impossible to neglect, unlike the 'rigid' impact described by the Newton elementary theory with coefficient of restitution, see Fig. 1(c). The transition from the linear oscillator (Fig. 1(a)) into oscillator with rigid impacts can be accomplished by means of this conception, when the stiffness $k_{2}$ changes in interval $0 \leqslant k_{2} \leqslant \infty$. The dynamics of both boundary cases is well known [4].

There exist a large variety of mathematical models of soft impacts (e.g. schemes in Fig. 2, where $F$ denotes the contact force in dependence on deformation of the soft stop) corresponding to real practical situation. Some of them were studied, e.g. the Kelvin-Voigt model (Fig. 2(a)) in [1], [12] or [2], the piecewise linear model (Fig. 2(b)) in [13,14] and the Hertz model (Fig. 2(c)) in [15,16].

Results obtained were generalised in [8] and the influence of the hardening of impacts ( $k_{2}$ increases from zero to infinity) can be expressed as it follows.

(1) The birth of non-linear phenomena on grazing boundary, where impacts appear in impact-less motion. No hysteresis regions exist for small values of the stop spring stiffness $k_{2}$ (Fig. 2(a) and (b)).

(2) Regions of subharmonic and chaotic impact motions become wider and develop from grazing boundary.

(3) Transition across grazing boundary from impact-less into impact motion is continuous and reversible for motion with soft impacts and very narrow region of impact motion with one weak impact per cycle exists along this boundary. Nevertheless hysteresis region of impact motion into impact-less motion region exists. It is caused by the existence of saddle-node stability boundary, where the regime with weak impact jumps into the same regime with stronger impact. Only this motion exhibits hysteresis phenomenon.

(4) The structure of fundamental and subharmonic impact motions does not depend considerably on the model of soft impact. 


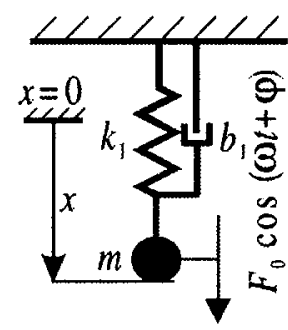

(a)


(c)

Fig. 1. Scheme of transition from linear motion: (a) through motion with soft impacts; (b) into motion with rigid impacts; (c) of one degree of freedom oscillator.

(a)

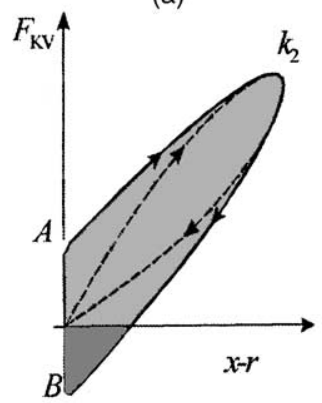

(b)

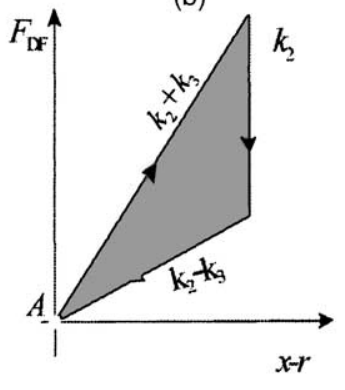

(c)

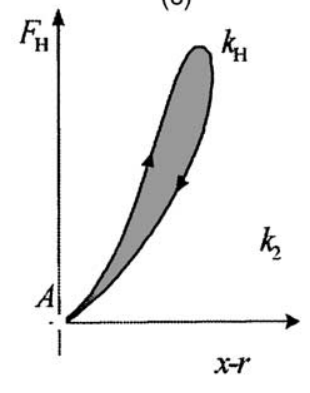

(d)

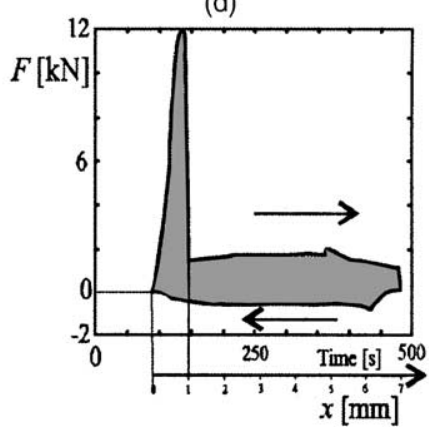

Fig. 2. Examples of force interactions during soft impacts.

(5) Regions of existence and stability of periodic subharmonic impact motions, beginning from a certain order, lie only over the grazing bifurcation boundary. Their appearance is conditioned by a special selection of initial conditions of the system motion.

One application of the oscillator with soft impacts is assumed in connection with the analysis of motion of a new type of piercing machine. The model of deformation and dissipative characteristics of forming can be complex (Fig. 2(d)), but a simple model of soft impact with preloaded stop is shown to present when the machine is not supplied by formed material. Then parts of the impact model (Fig. 2(d)) vanish and is characterized by a very small damping. The response of forming machine on the periodic excitation can be therefore very diverse [3]. The comparison of the system behaviour in working and discharged regime is introduced in the paper.

\section{Mathematical model of piercing forces and system motion}

The simulation of the piercing machine motion and the development of a model for soft impact interactions started from experimentally ascertained piercing and dry friction forces (Fig. 2(d)). They were accomplished by components corresponding to the design of a piercing tool (Fig. 3). It is prepared for the piercing of the chain link detail (Fig. 4) from the thin sheet strip. The tool is inserted between moving bodies of the double impact oscillator [7] and sheet strip is cut through by punches $1,2,3$ (Fig. 3).

The detail is moved back using additional pre-stressed springs $a, b$ and the push back 4 . The chain link motion is damped by friction force in cutting tool. 




Fig. 3. Scheme of tool.

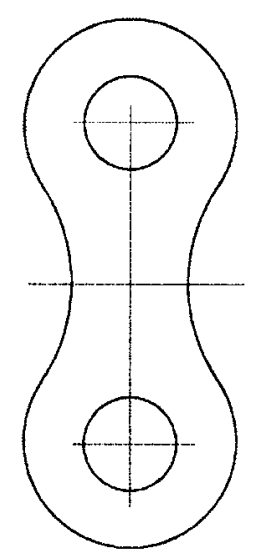

Fig. 4. Detail of a chain link.

Mathematical model of forming force $F$ is composed from several parts (Fig. 5) corresponding to the compression and tensile test (Fig. 2(d)) as well as additional springs $a, b$ and rigid impacts, when clearances in the tool are taken up. This model was reconstructed according to the piercing of the hole in the chain link.

Particular phases of mathematical model can be expressed in the form

Phase I

$$
F_{\mathrm{I}}=\frac{x-r}{k_{1}}\left(k_{2}+k_{3} \operatorname{sign} \dot{x}\right) \quad \text { for } r<x<r+s .
$$

Phase II

$$
F_{\text {II }+}=\frac{s}{10 k_{1}}\left(k_{2}+k_{3}\right)=\frac{s}{10 k_{1}}\left(F_{I}\right)_{\max } \text { for } x>r+s
$$




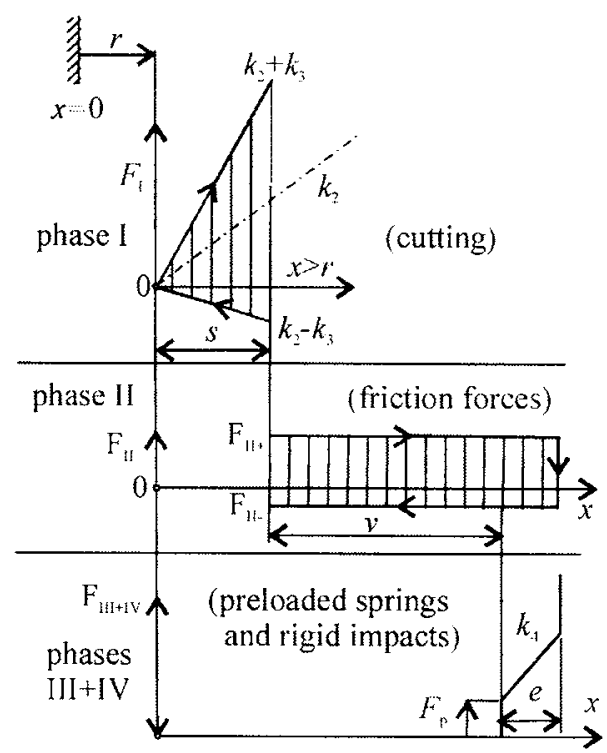

Fig. 5. Mathematical model of piercing forces.

$$
\begin{aligned}
& F_{\mathrm{II}-}=-\frac{s}{20 k_{1}}\left(k_{2}+k_{3}\right)=-\frac{s}{20 k_{1}}\left(F_{I}\right)_{\max } \text { for } x>r+s, \\
& F_{\mathrm{II}}=\frac{s}{40 k_{1}}\left(k_{2}+k_{3}\right)(1+3 \operatorname{sign} \dot{x}) .
\end{aligned}
$$

Phase III

$$
\begin{aligned}
& F_{\mathrm{III}}=F_{\mathrm{p}}+\frac{k_{4}}{k_{1}}(x-r-s-v) \text { for } r+s+v<x<r+s+v+e, \\
& F_{p} \doteq 2 F_{\mathrm{II}+} \doteq \frac{s}{5 k_{1}}\left(k_{2}+k_{3}\right) .
\end{aligned}
$$

Phase IV

$$
F_{\mathrm{IV}}=\infty, \quad \dot{x}_{+}=-R \dot{x}_{-} \quad \text { for } x=r+s+v+e,
$$

where $\dot{x}_{+}$and $\dot{x}_{-}$is post- and pre-impact velocity, respectively and $R$ is coefficient of restitution (the Newton model of impact).

Mathematical model of the impact oscillator (Fig. 1(b)) motion with forming force $F$ is described by equation

$$
m \ddot{x}+b_{1} \dot{x}+k_{1} x+F=F_{0} \cos (\omega t+\varphi) .
$$

It is solved using numerical simulation [5] after time $\tau=\Omega t$ and amplitude $X=x / x_{\text {st }}$ transformations, where $\Omega=\sqrt{k_{1} / m}$ is natural frequency of impact-less motion of the oscillator and $x_{\mathrm{st}}=F_{0} / k_{1}$ is static deflection of mass $m$. Results are shown in dependence on important dimensionless parameters $\eta=\omega / \Omega$ and $\rho=r / x_{\text {st }}$. Different regimes of impact motion are classified by quantity $z=p / n$, where $p$ is number of impacts and $n$ is number of excitation force periods $T=2 \pi / \omega$ in one period of impact motion. It means also mean number of impacts per one period $T$ for the chaotic motion.

\section{Bifurcation diagrams and motion trajectories}

There exist specific problems of the dynamics of impact oscillators, which can be explained by regions $(\eta, \rho)$ of existence of different regimes of the impact oscillator.

More detail information of the practical importance is contained in bifurcation diagrams along sections of mentioned regions and in time series and phase trajectories of motion in specific points of plane $(\eta, \rho)$. 
Fig. 6 shows motion amplitudes $X_{\mathrm{m}}(\eta)$ and before-impact velocities $X_{-}^{\prime}(\eta)$ of the impact motion (meaning of these quantities is shown in Fig. 7) for series values of dimensionless clearance $\rho=2,6,10, \ldots, 22$ in frequency interval $(0.8<\eta<1.8)$ for constant dimensionless parameters

$k_{2} / k_{1}=21, k_{3} / k_{1}=23, k_{4} / k_{1}=7$-stiffness of force $F$,

$\beta_{1}=b_{1} / 2 \sqrt{m k_{1}}=0.02$ - viscous damping of impact-less motion,

$\beta=b / 2 \sqrt{m k_{1}}=0.09$-damping of motion during connection of impacting bodies,

$\sigma=s / x_{\mathrm{st}}=0.4-$ width of pierced material,

$\sigma+v / x_{\mathrm{st}}=0.9$ - displacement between start of piercing and preloaded stop,

$\varepsilon=e / x_{\mathrm{st}}=5$ - displacement between start of the preloaded stop action and rigid stop (large value of $\varepsilon$ is assumed here for exclusion of rigid impacts),

$F_{\mathrm{II}+} / F_{0}=\sigma\left(k_{2}+k_{3}\right) /\left(10 k_{1}\right)=1.76, \quad F_{\mathrm{II}-} / F_{0}=-\sigma\left(k_{2}+k_{3}\right) /\left(20 k_{1}\right)=-0.88, \quad F_{\mathrm{p}} / F_{0}=10$ - constant $\quad$ values $\quad$ in force $F$.
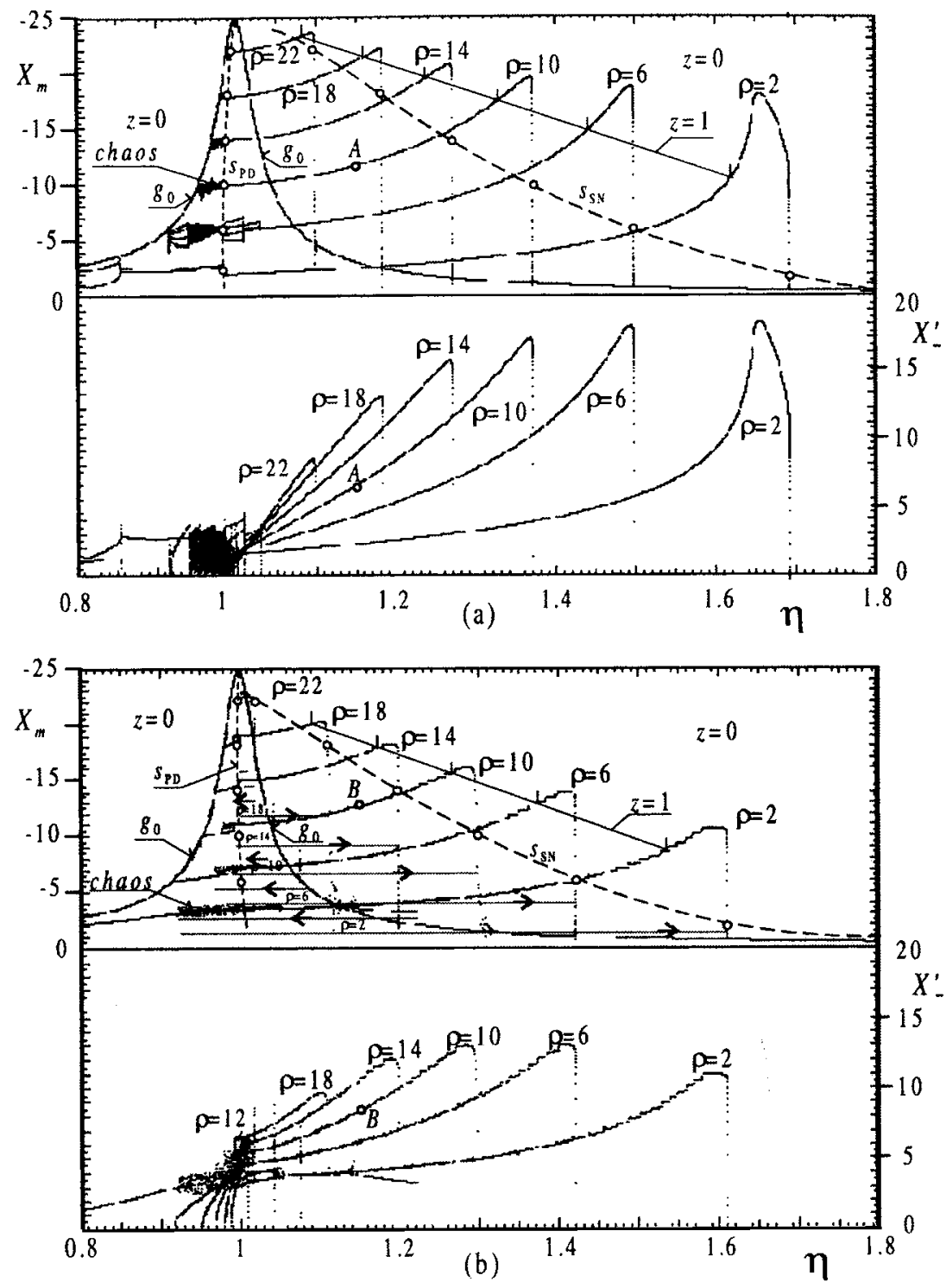

Fig. 6. Bifurcation diagrams of impact motion without (a) and with (b) piercing. 

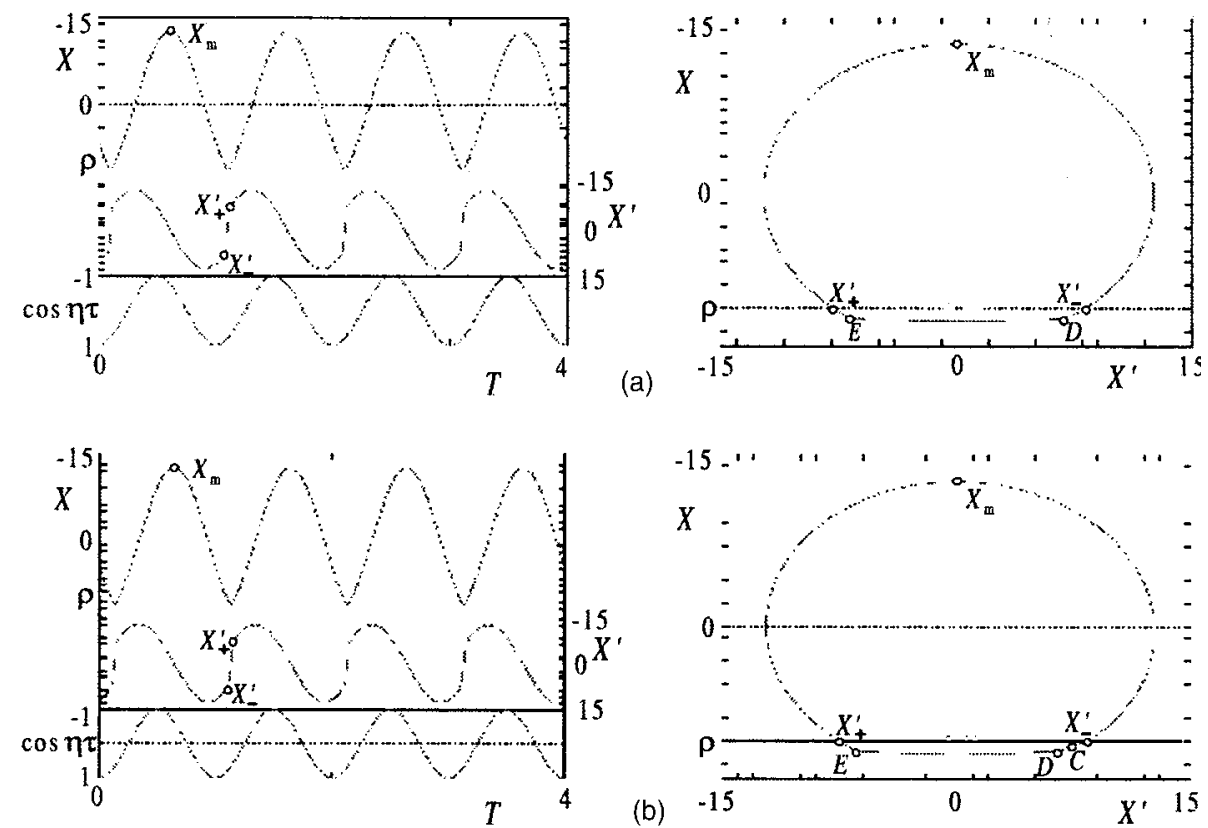

Fig. 7. Time series and phase trajectories of $z=1$ impact motion in points $A, B$ of Fig. 6 for unloaded (a) and working (b) regime, respectively.

Boundary $g_{0}$ in Fig. 6 represents the frequency response of linear oscillator to periodic excitation. It is simultaneously grazing bifurcation boundary, where impactless motion transits into regime with impacts. The impact motion should exist under boundary $g_{0}$, where amplitudes of linear motion are higher then clearance $\rho$.

The transition cross boundary $g_{0}$ is continuous under resonance of impact-less motion $(\eta<1)$ and different periodic subharmonic and chaotic impact motions appear $(0<z<1)$. These motions stabilise in $z=1$ motion on period doubling bifurcation boundary $S_{\mathrm{PD}}$. On the other hand $(1<\eta<2)$ periodic $z=1$ impact motion (Fig. 7) arises by jump from impact-less motion and is stable up to its saddle-mode bifurcation boundary $s_{\mathrm{SN}}$. This periodic regime is most important for a practical application and exists in large region between stability boundaries $s_{\mathrm{PD}}$ and $s_{\mathrm{SN}}$.

Hysteresis region between boundaries $g_{0}$ and $s_{\mathrm{SN}}$ therefore exists, where both impact-less and $z=1$ impact motion can exist in dependence on motion initial condition, direction of the system parameters changes, etc.

Amplitudes of the system motion increases with increasing clearance $\rho$ (Fig. 6), but there exist the optimum clearance $\rho$ for maximum before-impact velocities. Extreme amplitude $X_{\mathrm{m}}$ and before-impact velocity $X^{\prime}$ appear near stability boundary $s_{\mathrm{SN}}$. The motion stability measure, expressed e.g. by the Lyapunov exponent, has similar course [9], so the more intensive impact motion is more resistant against disturbances or is more stable, nevertheless the establishing of extreme regime from motion initial conditions is difficult. The establishing certainty of $z=1$ impact motion in hysteresis region increases from zero, on stability boundary $s_{\mathrm{SN}}$, to one on grazing boundary $g_{0}$. It is proved by the evaluation of basins of attraction [7]. It follows from this knowledge that the election of system parameters for practical application of $z=1$ impact motion should be a compromise among several aspects and will correspond to some point in the middle of hysteresis region, for example in points $A$ and $B(\eta=1.15, \rho=10)$ of Fig. 6. Motion trajectories in points A, B are shown in Fig. 7.

Figs. 6(a) and 7(a) correspond to the system motion with preloaded stop, without piercing, dry friction forces and rigid impacts. Figs. 6(b) and 7(b) show similar results for assumed model of piercing (without rigid impacts). Hysteresis region, motion amplitudes and before-impact velocities of regime with piercing are less in comparison with the regime without piercing. It can be expected that motion disturbances, caused by the intermittent interruption of the formed material supply, will not interrupt the regime of $z=1$ motion. Not all impact regimes are satisfactory strong for piercing. Motion with piercing is indicated in Fig. 6(b) by couple of line segments for every clearance $\rho$. Longer and shorter line corresponds to increasing and decreasing frequency $\eta$, respectively, which is also expressed by arrows on line segments. 
Small difference between working and unloaded regime is also shown on phase trajectories in Fig. 7. Sections $D E$ correspond to impacts with preloaded stop. Section $X_{-}^{\prime} C$ and $C D$ in Fig. 7(b) corresponds to piercing and motion with dry friction forces, respectively.

\section{Evaluation of bifurcation diagram}

The dynamics of motion with rigid and soft impacts was explained in $[4,7,8,12]$, respectively. The motion with preloaded stop represents the intermediate case, when impact force is discontinuous, similar as in rigid impacts and depends on the stop deformation, as in soft impacts. The more detail investigation of such type of impact oscillator is therefore important also from the point of view of the basic research.

The behaviour of this system will be shown on bifurcation diagram for clearance $\rho=10$ in Fig. 6(a). Results are presented in Fig. 8 and Table 1 in the form of evaluation of quantity $z$ of all obtained regimes of the system motion. They are supplemented by time series, phase trajectories and Poincaré maps shown in Fig. 9 (Fig. 9(A)-(L) correspond to frequencies (A)-(L) marked in Fig. 8). Four subintervals (a)-(d) are selected from frequency interval $0.8<\eta<1.8$. There appear bifurcations (1)-(19) marked in Fig. 8 and Table 1 and characterized by abbreviations, the meaning of which is determined in Table 2 .

Changes appearing during bifurcations are characterised also by arrows in Fig. 8. Unidirectional arrow expresses the inreversible change of the system motion, e.g. change connected with the existence of hysteresis. Bi-directional arrows correspond to reversible transition, without hysteresis. All bifurcations and transitions between periodic and chaotic

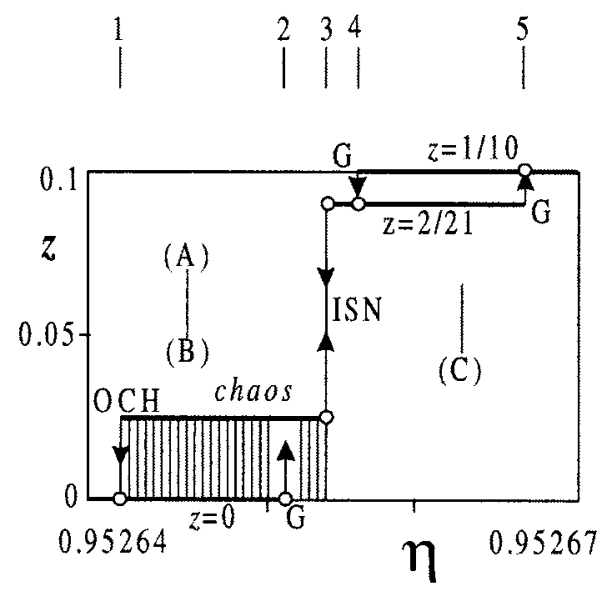

(a)



(b)

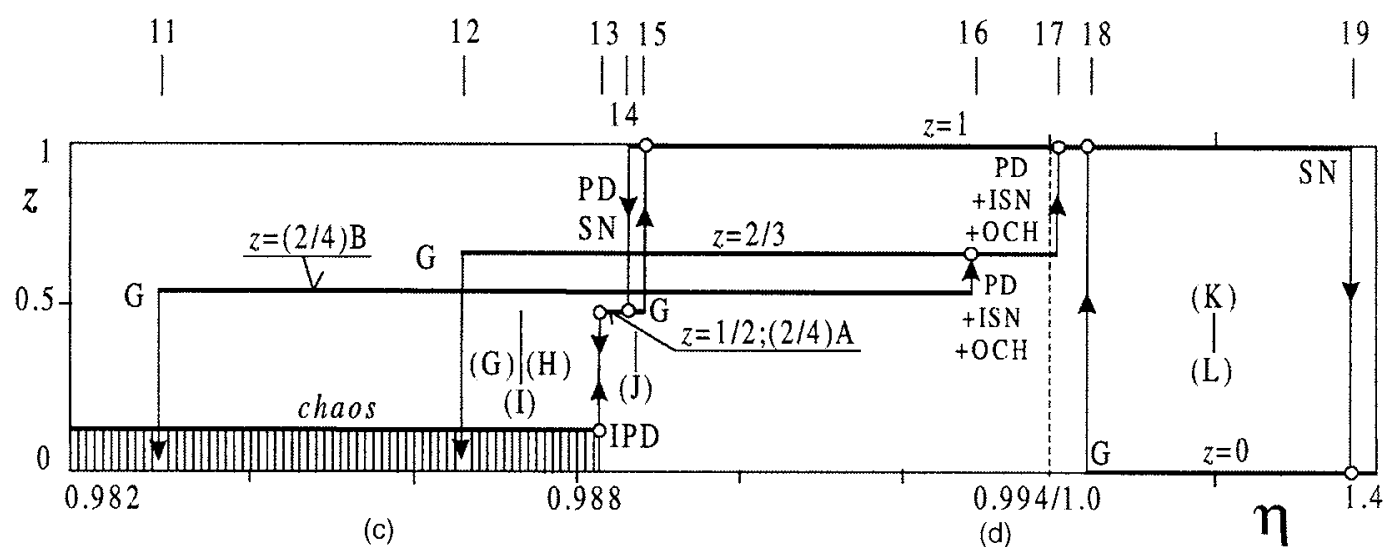

Fig. 8. Bifurcation diagram along curve $\rho=10$ in Fig. 6(a). 
Table 1

Characteristic of bifurcations in Fig. 8

\begin{tabular}{|c|c|c|c|c|c|}
\hline & No. & $\eta$ & Transition & hyst & Type \\
\hline \multirow[t]{5}{*}{ (a) } & 1 & 0.95264175 & chaos $\rightarrow z=0$ & Yes & $\mathrm{OCH}$ \\
\hline & 2 & 0.95265156 & $z=0 \rightarrow$ chaos & Yes & G \\
\hline & 3 & 0.95265475 & chaos $\leftrightarrow z=2 / 21$ & No & ISN \\
\hline & 4 & 0.95265672 & $z=1 / 10 \rightarrow z=2 / 21$ & Yes & G \\
\hline & 5 & 0.95266678 & $z=2 / 21 \rightarrow z=1 / 10$ & Yes & G \\
\hline \multirow[t]{5}{*}{ (b) } & 6 & 0.95341005 & chaos $\rightarrow z=1 / 10$ & Yes & $\mathrm{OCH}$ \\
\hline & 7 & 0.95380362 & $z=1 / 10 \rightarrow$ chaos & Yes & G \\
\hline & 8 & 0.95406 & chaos $\rightarrow z=1 / 9$ & No & IPD \\
\hline & 9 & 0.95542 & $\operatorname{chaos} \rightarrow z=1 / 9$ & Yes & $\mathrm{OCH}$ \\
\hline & 10 & 0.95615 & $z=1 / 9 \rightarrow$ chaos & Yes & G \\
\hline \multirow[t]{6}{*}{ (c) } & 11 & 0.98305 & $z=(2 / 4) \mathrm{B} \rightarrow$ chaos & Yes & G \\
\hline & 12 & 0.98678 & $z=2 / 3 \rightarrow$ chaos & Yes & G \\
\hline & 13 & 0.98849 & chaos $\leftrightarrow z=(2 / 4) \mathrm{A}$ & No & IPD \\
\hline & 14 & 0.98890 & $z=1 \rightarrow z=1 / 2$ & Yes & $\mathrm{PD} \rightarrow \mathrm{SN}$ \\
\hline & 15 & 0.98899 & $z=(2 / 4) \mathrm{A} \rightarrow z=1$ & Yes & G \\
\hline & 16 & 0.99273 & $z=(2 / 4) \mathrm{B} \rightarrow(4 / 8) \mathrm{B} \rightarrow$ chaos $\rightarrow z=2 / 3$ & Yes & $\mathrm{PD} \rightarrow \mathrm{ISN} \rightarrow \mathrm{OCH}$ \\
\hline \multirow[t]{3}{*}{ (d) } & 17 & 1.00544 & $z=2 / 3 \rightarrow 4 / 6 \rightarrow$ chaos $\rightarrow z=1$ & Yes & $\mathrm{PD} \rightarrow \mathrm{ISN} \rightarrow \mathrm{OCH}$ \\
\hline & 18 & 1.04444 & $z=0 \rightarrow z=1$ & Yes & G \\
\hline & 19 & 1.37083 & $z=1 \rightarrow z=0$ & Yes & $\mathrm{SN}$ \\
\hline
\end{tabular}

Table 2

Characteristic of bifurcation

\begin{tabular}{ll}
\hline Bifurcation & Characterization \\
\hline $\mathrm{G}$ & Grazing-touch of impact-less loop of motion with the stop \\
$\mathrm{SN}$ & Saddle-node instability and jump transition to another periodic motion \\
$\mathrm{PD}$ & Period doubling of impact motion \\
ISN & Interruption of saddle-node instability process and appearance of intermittency chaos \\
$\mathrm{IPD}$ & Interruption of Faigenbaum's cascade of period doublings and transition into chaos \\
$\mathrm{OCH}$ & output from chaotic into periodic motion of the system \\
$\mathrm{PD} \rightarrow \mathrm{SN} \mathrm{PD} \rightarrow$ ISN $\rightarrow$ OCH & Chronological succession of bifurcations which appear after very slight change of \\
& frequency $\eta$ \\
\hline
\end{tabular}

impact motions are explained in [6,10] for oscillator with rigid impacts. This knowledge can be used for explanation of bifurcations in Figs. 6 and 8.

The typical feature of the system motion, corresponding to motion with soft impacts is the existence of motion $z=(2 / 4)$ B (Fig. 9(H)) and motion $z=2 / 3$ (Fig. 9(I)), which cannot be reached by successive, and quasistationary changes of parameters $\eta, \rho$ from impact-less $(z=0)$ motion. They belong to impact motion with higher level of kinetic energy. When motion $z=(2 / 4) \mathrm{B}$ is reached, then motion $z=2 / 3$ can be settled from it by quasistationary transition (bifurcations (16) in Fig. 8).

There are several other interests in the considered region of subharmonic and chaotic motions $(0.95<\eta<1)$ :

- the existence of ambiguousness of system response (Figs. 8 and 9 and motions (A) and (B) for $\eta=0.9526475$, motions (D) and (E) for $\eta=0.95365$ and motions (G), (H) and (I) for $\eta=0,9875$ ),

- two possible motions $z=(2 / 4)$ A and $z=(2 / 4)$ B (Fig. 9(J) and (H)), which differ by the position of impacts marked by arrows in the motion period (sequences of impacts in consecutive excitation periods $T$ are: 1-0-1-0 in Fig. 9(J) for regime A and 1-1-0-0 in Fig. 9(H) for regime B),

- the existence of complex periodic motions $z=2 / 21, z=1 / 10, z=1 / 9$ (Fig. 9(C), (D) and (F) near grazing bifurcation boundary $g_{0}$.

- $\mathrm{PD} \rightarrow \mathrm{SN}$ (for $\eta=0.9889$-bifurcation 14 in Fig. 8 and Table 1) is the splitting of $z=1$ to $z=2 / 2$ motion, which loses its stability on saddle-node bifurcation boundary. One impact in motion period disappears and $z=1 / 2$ motion stabilises (Fig. 9(J)), 


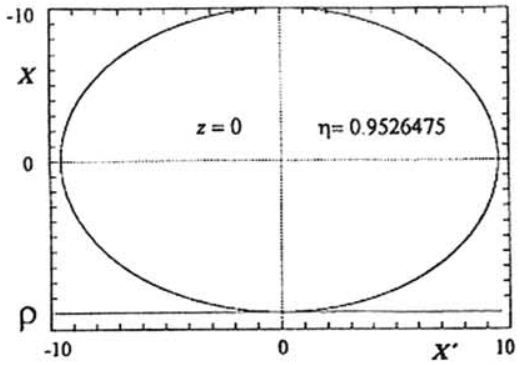

(A)

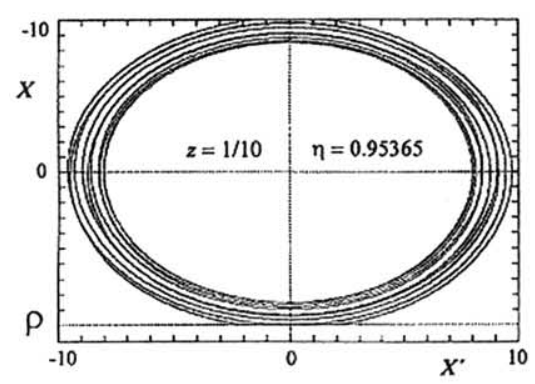

(D)

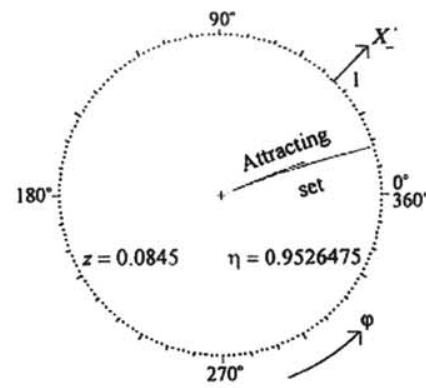

(B)

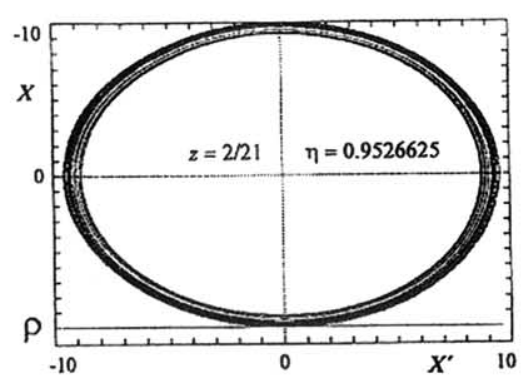

(C)



(E)



(F)

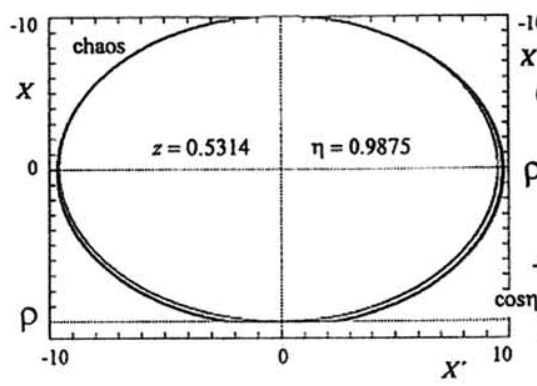

(G)

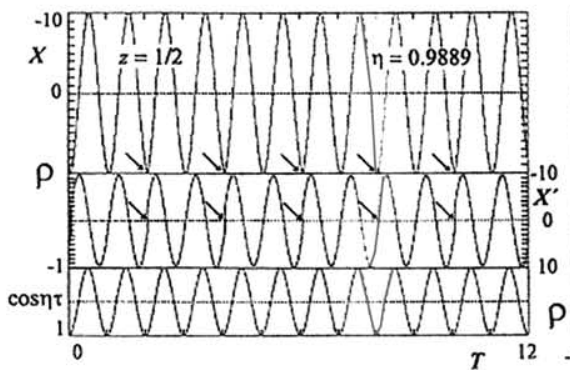

(J)

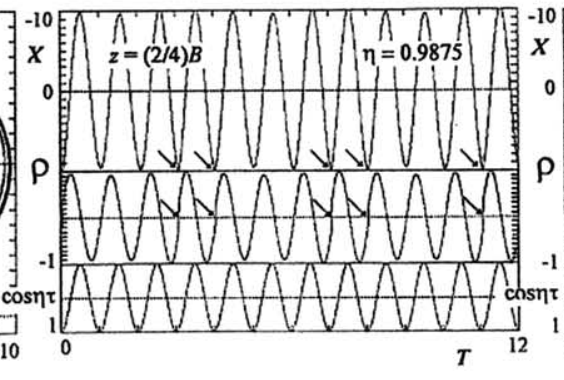

(H)

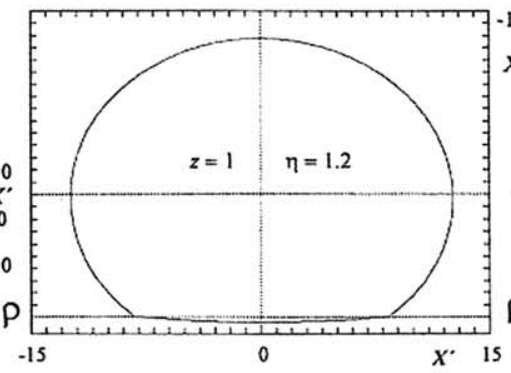

(K)

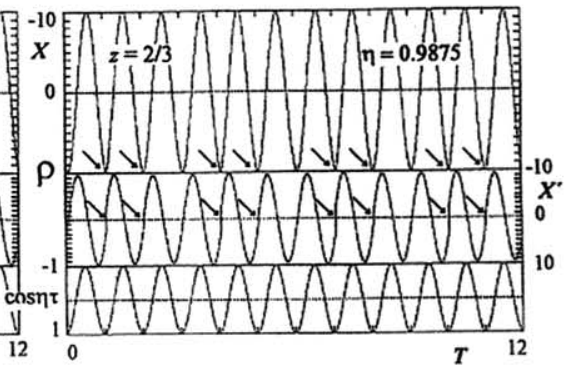

(I)

Fig. 9. Examples of different impact motions received by numerical simulation.

- $\mathrm{PD} \rightarrow \mathrm{ISN} \rightarrow \mathrm{OCH}$ (for $\eta=0.99273$ and 1.00544 bifurcation 16 and 17, respectively, in Fig. 8 and Table 1) are similar processes as described bifurcation $\mathrm{PD} \rightarrow \mathrm{SN}$. 
Nevertheless the jump transition (Fig. 9), i.e. the saddle-node instability development, cannot be accomplished due to the appearance of an additional impact, which interrupts this process (ISN) and causes to happen the reversible transition into the intermittency chaos [11]. The system motion escapes later from chaotic motion into periodic impact motion $(\mathrm{OCH})$, which is irreversible.

\section{Conclusion}

The application of the analysis of strongly non-linear systems with impacts into the field of forming machines requires the selection of a proper model of impact interactions. The question of response of the piercing machine on disturbances caused by the intermittent interruption of the formed material supply during the work of machine will not terminate the working regime. The work without piercing leads to the impact model characterized by preloaded stop and small damping. Many different periodic and chaotic impact regimes exist therefore in a narrow frequency interval. Bifurcations and transition phenomena between them were explained. Nevertheless there is large region of system parameters, where practically most impact motion regime appears. Parameters for obtaining maximum before-impact velocities were ascertained. An intermittent interruption of the forming material supply does not change the optimal working regime.

\section{Acknowledgements}

This investigation was financially supported by the Grant Agency of the Czech Republic, Project 101/00/0007 and by the Ministry of Education, Youth and Sport of the Czech Republic, Project COST P-4, OC P4.10.

\section{References}

[1] Hunt KH, Crossley FRE. Coefficient of restitution interpreted as damping in vibro-impact. JAM, Trans ASME 1975:440-5.

[2] Kocanda L. Theoretical analysis of soft impact oscillator. In: Book of abstracts EUROMECH 425 colloquium on nonlinear dynamics control and condition monitoring, August 20-24, 2001, Aberdeen, Scotland.

[3] Kotera T, Peterka F. Laws of impact motion of mechanical systems with one degree of freedom. Part VI—analytical and analogue simulation of the multi-impact motion and its stability. Acta Techn. ČSAV 1984;3:255-79.

[4] Peterka F. Introduction to vibration of mechanical systems with internal impacts. Academia 1981:269 [in Czech].

[5] Peterka F. Problems of numerical simulation of the impact oscillator motion. Eng Mech 1998;5(2):71-87.

[6] Peterka F. Dynamics of the impact oscillator. In Moon FC editor, Proceedings of IUTAM symposium new applications of nonlinear and chaotic dynamics in mechanics, Ithaca, 27 July-1 August, 1997. Dordrecht: Kluwer Academic Publishers; 1999. p. 283-92.

[7] Peterka F. Global analysis of the double impact oscillator dynamics. In: Book of abstracts ASME DETC'99, Las Vegas, USA, September 12-16, 1999, proceedings of CD-ROM, paper DETC 1999/VIB-8039; 1999.

[8] Peterka F. Dynamics of oscillator with soft impacts. In: Proceedings of ASME DETC'01, September 9-12, 2001, Pittsburgh, USA, CD-ROM, paper DETC 2001/VIB-21609 2001.

[9] Peterka F, Čipera S. Chaotic motions in mechanical systems with impacts. In: Kapitaniak T, Brindley J editors. Proceedings of chaos and nonlinear mechanics. Leon OC editor. Series B, vol. 4. Singapore: World Scientific; 1994. p. $265-76$.

[10] Peterka F, Kotera T. Four ways from periodic to chaotic motion in the impact oscillator. Machine Vibrat 1996;5:71-81.

[11] Peterka F, Kotera T, Ćpera S. Explanation of appearance and properties of intermittence chaos in the impact oscillator dynamics. In: Proceedings of ASME DETC'01, September 9-12, 2001, Pittsburgh, USA, CD-ROM, paper DETC 2001/VIB-21440 2001.

[12] Peterka F. Szöllös O. Influence of the stop stiffness on the impact oscillator dynamics. In: Proceedings of IUTAM symposium on unilateral multibody dynamics, München, 3-7 August, 1998. Dordrecht: Kluwer Academic Publishers; 1999 p. $127-35$.

[13] Peterka F, Tondl A. Dynamics of oscillator with piecewise linear model of soft impacts. In: Book of abstracts, engineering mechanics 2001. Czech Republic: Svratka; 2001a. p. 209-10 [Proceedings of CD-ROM, Institute of Thermomechanics AS CR].

[14] Peterka F, Tondl A. Dynamics of oscillator with piecewise linear model of impact interactions. In: Proceedings of the colloquium dynamics of machines 2001. Prague: Institute of Thermomechanics AS CR; 2001. p. 161-6.

[15] Půst L, Peterka F. Impact oscillator with Hertz's model of contact. In: Book of abstracts EUROMECH 425 colloquium on nonlinear dynamics control and condition monitoring, August 20-24, 2001, Aberdeen, Scotland; $2001 \mathrm{a}$.

[16] Půst L, Peterka F. Impact oscillator with the Hertz contact. In: Proceedings of colloquium dynamics of machines 2001. Prague: Institute of Thermomechanics AS CR; 2001. p. 167-74. 